\title{
Transmission of Hypervirulent Acinetobacter baumannii Between General and Intensive Care Unit
}

Min Zhang

Shenzhen People's Hospital

Jinyong Zhang

Army Medical University

Shiyi Liu

Shenzhen People's Hospital

Yuxin Zhong

Shenzhen People's Hospital

Yuemei Lu

Shenzhen People's Hospital

Jinsong Wu

Shenzhen People's Hospital

Weiyuan Wu

Shenzhen People's Hospital

Yingyun Fu

Shenzhen People's Hospital

Lingwei Wang

Shenzhen People's Hospital

Dandan Chen

Shenzhen People's Hospital

Chuchu Lin

Shenzhen People's Hospital

Wenping Zheng

Shenzhen People's Hospital

Chen Qiu

Shenzhen People's Hospital

Quanming Zou

Army Medical University

Wei Huang ( $\nabla$ whuang_sz@163.com )

Shenzhen People's Hospital https://orcid.org/0000-0002-8569-5184 
Keywords: Acinetobacter baumannii, outbreak, hypervirulent, transmission

Posted Date: January 7th, 2020

DOI: https://doi.org/10.21203/rs.2.20225/v1

License: (c) (1) This work is licensed under a Creative Commons Attribution 4.0 International License. Read Full License 


\section{Abstract}

Background Acinetobacter baumannii is one of the most important microorganisms associated with hospital-acquired infections and nosocomial outbreaks.Aim We investigate a hypervirulent A. baumannii outbreak with aim to identify the transmission mediator.

Methods We investigated an outbreak in respiratory intensive critical care unit (RICU) and respiratory medicine unit (RMU) based on whole genome sequencing (WGS) and epidemiology data. Virulence potential was performed in a mouse model.

Results Eight and two CRAB isolates were isolated in RICU and RMU during August 2018. Four fatal cases were all involved in a transmission event. In vivo test confirmed the hypervirulence phenotype of the isolates. We inferred that the portable devices were probably the mediator of the transmission based on the transmission chain and epidemiology data. We isolated an hypervirulent CRAB from the screen of portable ventilator. Portable devices disinfection using vaporized hydrogen peroxide and avoiding the sharing of devices between general ward and ICU successfully blocked the further transmission.

Conclusions An emerging hypervirulent A. baumannii with enhanced virulence has successfully colonized on the surface of mobile medical device. Sharing these mobile devices may lead to spread between the general ward and ICU. The new transmission chain construction tool is useful to the outbreak investigation.

\section{Introduction}

Acinetobacter baumannii is one of the most important microorganisms associated with hospital-acquired infections worldwide [1]. In intensive care units (ICUs), A. baumannii account for up to $20 \%$ of infections and manifest as ventilator-associated pneumonia and central-line-associated bloodstream infections [2, 3].

Spread of A. baumannii between patients in hospital settings is difficult to control because of their ability to persist in environments such as computer keyboards, pillows, curtains and other dry surfaces, which necessitates strict adherence to infection control measures [4]. Numerous nosocomial outbreaks of $A$. baumannii have been reported [5].

In general, A. baumannii has been regarded as a low-grade pathogen. Most laboratory strains and clinical isolates do not cause severe infections in immunocompetent mice, inducing only a self-limiting pneumonia with very limited local bacterial replication and systemic dissemination, even when a large inoculum is used $[6,7]$. However, studies have shown that some clinical carbapenem-resistant A. baumannii (CRAB) strains are lethal to immunocompetent mice and thus revealed the range of virulence in different strains of this pathogen $[8,9]$. 
In this study, we retrospectively investigated an outbreak of CRAB and confirmed the transmission of hypervirulent A. baumannii between general ward and ICU.

\section{Methods}

\section{Outbreak investigation}

The incidence rate of CRAB in respiratory intensive critical care unit (RICU) was 3.4 cases per month in Shenzhen people's hospital in 2017. However, we isolated 8 isolates in August 2018 and therefore initiated an outbreak investigation. Given the frequent transfer of patients between the RICU and respiratory medicine unit (RMU), we also collected 2 CRAB isolates from RMU in the same period.

\section{Phenotypic characterization}

We used a VITEK-2 compact system (bioMérieux, Marcy-l'Étoile, France) to establish the identity and antimicrobial susceptibilities of the isolates. We interpreted the results in accordance with the guidelines published by the Clinical and Laboratory Standards Institute (CLSl; document M100-S26) [10]. CRAB was defined as resistance to imipenem or meropenem.

\section{WGS and identification}

Genomic DNA of isolates was extracted using the Sodium Dodecyl Sulfate (SDS) method and quantified with a Qubit (Thermo Fisher, Waltham, MA, USA). The DNA library was constructed with an insert size of $350 \mathrm{bp}$. A-tailed, paired-end adapters were ligated into the DNA fragments and sequenced on an Illumina HiSeq 2500 platform (Illumina, San Diego, CA, USA). Quality control, preprocessing of fastq files, trimming of adaptor, low-quality (phred quality $<$ Q15), low complexity reads $(30 \%)$, and polyG/polyX tails were performed using fastp [11]. Genome assembly was performed using the de novo SPAdes Genome Assembler (version 3.12.0) [12]. We scanned genome contigs against the ResFinder database with Staramr (version 0.2.2) to detect antimicrobial resistance genes in the isolates [13]. We annotated the results with Prokka (version 1.13.3) [14]. Multilocus sequence typing (MLST) was performed with Ridom SeqSphere ${ }^{+}$(version 5.1.0) [15].

\section{Transmission trees construction}

Alignment and single nucleotide polymorphism (SNPs) extraction from assembly contigs was performed using the Harvest suite (version 1.2) [16]. Transmission trees were constructed based on single nucleotide polymorphism (SNPs) and integrated epidemiological information (i.e., patient exposure to infection) with 
the BEAST2 (version 2.5.1) package Structured Coalescent Transmission Tree Inference (SCOTTI) (version 1.1.1) [17]. SCOTTI not only accounts for diversity and evolution within a host, but also for other sources of bias namely non-sampled hosts and multiple infections of the same host. This new method builds on recent progress in efficiently modeling migration between populations using an approximation to the structured coalescent. SCOTTI also uses epidemiological data about host exposure time, only allowing hosts to transmit the disease during periods when they are infectious [17]. We showed transmissions with a probability $>20 \%$.

\section{Virulence potential test}

A pneumonia model of $A$. baumannii in mice was used to test the virulence. Specific-pathogen-free grade C57BL/6 6-8 weeks old female mice were purchased from Hunan SJA laboratory animal CO., LTD (Hunan, China). The mice were intraperitoneally anaesthetized with pentobarbital sodium $(75 \mathrm{mg} / \mathrm{kg})$, then inoculated with $20 \mu \mathrm{L}\left(2.5 \times 10^{8}\right.$ and $\left.5 \times 10^{7} \mathrm{CFU}\right)$ of $A$. baumannii by non-invasive intratracheal instillation under direct vision. Mouse survival was observed for 7 days post infection. All animal care and use protocols in this study were performed in accordance with the Regulations for the Administration of Affairs Concerning Experimental Animals approved by the State Council of the People's Republic of China.

\section{Results}

\section{Phenotype and genotype of cases in this study}

In this study, 8 non-repetitive CRAB isolates were recovered from separate patients (case 1 to 8,6 from bronchoalveolar lavages and 2 from sputum) in August 2018. We also collected 2 CRAB isolates (case 9 to 10,1 from sputum and 1 from urine) from RMU in the same period. All isolates were assigned to ST2 with the Pasteur scheme, and b/a $a_{0 \times-23}$ was the only carbapenemase gene detected in this study (Figure 1). All A. baumanni isolates were resistant to both imipenem and meropenem.

\section{Transmission network}

We integrated epidemiological and WGS data to infer the transmission chain by SCOTTI. Three transmission events were identified, one of which was highlighted because all the fatal cases (case 3, 5, 8 and 10) occurred during this event. From the transmission network of this event, case 3 was considered the root of transmission. Case 5 was infected followed by case 3; Case 9 was infected followed by case 5; Case 10 was infected followed by case 8 . Two possibilities were inferenced of the source of case 8 (Figure 2). 
We collected the history of patients with the goal of identifying the mediator of transmission with fatal cases. The host of case 5 stayed in the RMU between July 15 and 29 and was transferred to the RICU until September 12. Four days after the transference of case 5, case 9 was hospitalized in RMU and stayed in the same bed. Case 3 and 8 stayed in the RICU from July 31 to August 12 and July 24 to August 23 respectively, while case 10 stayed in the RMU from August 11 to 26 . A spatial interaction in the RICU was found between case 3 and 8 , case 5 and 8 , and case 3 and 5 . No spatial interaction was observed between case 8 and 9 or 10 (Figure 3).

The RMU and RICU were located in the same building in the hospital and shared one bedside chest roentgenogram machine and bronchofibroscope room. The history of medical examination showed that all of the cases received the bedside chest roentgenogram before infection. In addition, case 5 and 8 received bronchofibroscope examination before infection (Figure 3).

Combining the epidemiological data and transmission network, because case 5 and 9 had been hospitalized in RMU and stayed in the same bed, we considered the spatial interaction and sharing of bedside chest roentgenogram machine may be the cause of transmission between case 3, 5, 8 and 9 . Transmission by health care workers was less likely because these patients belong to different treatment groups. Bedside chest roentgenogram to be the likely mediator of transmission between case 8 and 10 because no other relevant link could be established between them (Figure 3).

\section{Object surface sampling and intervention}

Hereby, we performed object surface sampling of all mobile medical equipment for public use in multiple departments including bedside bronchofibroscope, portable ventilator, transport stretcher, and bedside chest roentgenogram. We isolated the Bacillus cereus, Staphylococcus haemolyticus and Bacillus pumilus from the screen of bedside chest roentgenogram. Acinetobacter /woffii was isolated from the placement plate of bedside chest roentgenogram. Notably, we isolated an A. baumannii from the screen of portable ventilator.

We then disinfected all portable devices using vaporized hydrogen peroxide in a special confined space [18]. At the same time, we designated personnel responsible for the periodic disinfection of portable devices. Mobile devices in the ICU are no longer shared with general wards. Retrospective data showed that after regular disinfection and management of mobile devices, compared to the same period in 2018, the detection rate of multi-drug resistant bacteria in our hospital halved between January and December 2019.

\section{Virulence test}


Genomic analysis showed that the isolates involved in fatal transmission and from the screen of portable ventilator were all sharing the same K-locus (KL49) to the hypervirulent strain LAC-4 [19]. We therefore selected isolate from case 3 , case 7 and the screen of portable ventilator and used a pneumonia model to test the virulence. LAC- 4 was used as hypervirulent control. Using an inoculum of $2.5 \times 10^{8}$ colony-forming unit (CFU), the survival of animals infected isolates from case 3 , the screen and LAC-4 at 7 days was $10 \%$, $10 \%$ and $0 \%$, while that of isolates from case 7 and ATCC 17978 was $70 \%$ and $90 \%$, respectively $(n=10$, $\mathrm{p}<0.0001$ by log-rank test) (Figure 4).

\section{Discussion}

A. baumannii is an opportunistic nosocomial infection pathogen that usually causes septicemia and pneumonia and outbreaks in ICU [20]. The isolation of an hypervirulent A. baumannii from mobile medical devices suggesting that this threatening pathogen has been successfully disseminated and colonized in the hospital.

For outbreak investigation, the finest scale is to ask whether direct transmission happened from a given host to another. WGS can provide a high-resolution tool to identify infection sources and augment efforts to prevent and eradicate infections. Integrated analysis of both epidemiological and sequence data clearly would maximize our ability to reconstruct transmission trees [21-24]. Several methods generally make use of the date of sampling, the interval of exposure of a host to an outbreak, the likely duration of infectiousness, or the within-host variation have emerged in recent years [25-30]. These approaches can generally infer transmission events even in the presence of considerable within-host variation, can account for the uncertainty associated with the possible presence of non-sampled hosts. As a result, inferring transmission between hosts with accuracy is becoming feasible. Recent years have seen progress in the development of statistically rigorous frameworks to infer outbreak transmission trees ("who infected whom") from epidemiological and genetic data [30].

In this study, we used an inference tool based on a Bayesian structured coalescent approximation route to reconstruct transmission within a CRAB outbreak. We found that the outbreak consists of several different transmission events. We identified the transmission event between host without spatial interaction. In this case, we can track the neglected mediator of transmission through the transmission chain and benefit the nosocomial infection control.

Our study highlights the role of mobile medical devices in the transmission of A. baumannii. We found that an emerging hypervirulent A. baumannii with enhanced virulence was successfully colonized on the surface of mobile medical device. Sharing these mobile devices may lead to spread between the general ward and ICU.

Vaporized hydrogen peroxide in a special confined space is a useful tool for the mobile medical device disinfection. Avoiding the sharing of devices between general ward and ICU and assigning personnel to disinfect portable devices regularly are the effective ways to prevent such transmission. 


\section{Conclusion}

In summary, we analyzed an outbreak of nosocomial infection caused by hypervirulent A. baumannii using transmission tree inference tool combined with epidemiological data. The analysis shows that the hyperviulent CRAB has been successfully disseminated and colonized in the hospital. Improper use of mobile devices can cause transmission between general wards and ICU. Therefore, it is necessary to regularly sterilize mobile devices and assign personnel to manage them. The new transmission analysis tool is useful to identify the transmission between the patients without spatial interaction.

\section{Declarations}

\section{Ethics approval and consent to participate}

All animal experiments in this study were approved by the Animal Ethical and Experimental Committee of the Army Military Medical University (Chongqing, Permit No. 2011-04) in accordance with their rules and regulations.

\section{Consent for publication}

Not applicable.

\section{Availability of data and materials}

Sequenc data that support the findings of this study have been deposited in GenBank with the accession codes PRJNA510897 and PRJNA546440.

\section{Competing interests}

The authors declare that they have no competing interests.

\section{Funding}

This work was supported by a grant from the Shenzhen Science and Technology Innovation Commission (grant numbers, GJHZ20180413181716797 and JCYJ20180305163929948). The funders played no role in the study design, data collection, analysis or decision to publish the work. 


\section{Authors Contribution}

ZM, LSY and ZYX performed data analysis. LCC, ZWP, LYM, WJS and WWY collected the isolates and identified the phenotype. FYY, WLW, CDD and QC collected the medical history. ZJY performed in vivo test. ZQM and HW were major contributors in writing the manuscript. All authors read and approved the final manuscript.

\section{Acknowledgements}

Not applicable.

\section{References}

1. Talbot GH, Bradley J, Edwards JE, Gilbert D, Scheld M, Bartlett JG\& Antimicrobial Availability Task Force of the Infectious Diseases Society of America. Bad bugs need drugs: an update on the development pipeline from the Antimicrobial Availability Task Force of the Infectious Diseases Society of America. Clin Infect Dis 2006; 42: 657-668.

2. Weiner LM, Webb AK, Limbago B, Dudeck MA, Patel J, Kallen AJ, et al. Antimicrobial-resistant pathogens associated with healthcare-associated infections: summary of data reported to the National Healthcare Safety Network at the Centers for Disease Control and Prevention, 2011-2014. Infect Control Hosp Epidemiol 2016; 37: 1288-301.

3. Vincent JL, Rello J, Marshall J, Silva E, Anzueto A, Martin CD, et al. International study of the prevalence and outcomes of infection in intensive care units. JAMA 2009; 302: 2323-2329.

4. Dijkshoorn L, Nemec A, Seifert H. An increasing threat in hospitals: multidrug-resistant Acinetobacter baumannii. Nat Rev Microbiol 2007; 5: 939-951.

5. Peleg AY, Seifert H, Paterson DL. Acinetobacter baumannii: emergence of a successful pathogen. Clin Microbiol Rev 2008; 21: 538-582.

6. van Faassen H, Kuo Lee R, Harris G, Zhao X, Conlan JW, Chen W. Neutrophils play an important role in host resistance to respiratory infection with Acinetobacter baumannii in mice. Infect Immun 2007; 75: 5597-5608. 区

7. Knapp S, Wieland CW, Florquin S, et al. Differential roles of CD14 and toll-like receptors 4 and 2 in murine Acinetobacter pneumonia. Am J Respir Crit Care Med 2006; 173: 122-129.

8. Zeng X, Gu H, Cheng Y, Jia KR, Liu D, Yuan Y, et al. A lethal pneumonia model of Acinetobacter baumannii: an investigation in immunocompetent mice. Clin Microbiol Infect 2019; 25:

9. Harris G, Kuo Lee R, Lam CK, Kanzaki G, Patel GB, Xu HH, et al. A mouse model of Acinetobacter baumannii-associated pneumonia using a clinically isolated hypervirulent strain. Antimicrob Agents Chemother 2013; 57: 3601-3613. 
10. Performance standards for antimicrobial susceptibility testing; twenty-sixth. informational supplement. CLSI document M100-S26. Clinical and Laboratory Standards Institute: Wayne, PA, 2016.

11. Chen S, Yanqing Z, Yaru C, Jia G. fastp: an ultra-fast all-in-one FASTQ preprocessor. Bioinformatics 2018; 34: 884-890.

12. Antipov D, Korobeynikov A, McLean JS, Pevzner PA. hybridSPAdes: an algorithm for hybrid assembly of short and long reads. Bioinformatics 2016; 32: 1009-1015.

13. Zankari E, Hasman H, Cosentino S, Vestergaard M, Rasmussen S, Lund O, et al. Identification of acquired antimicrobial resistance genes. J Antimicrob Chemother 2012; 67: 2640-2644.

14. Seemann T. Prokka: rapid prokaryotic genome annotation. Bioinformatics2014; 30: 2068-2069.

15. Jünemann S, Sedlazeck FJ, Prior K, Albersmeier A, John U, Kalinowski J, et al. Updating benchtop sequencing performance comparison. Nat Biotechnol2013; 31: 294-296.

16. Treangen TJ, Ondov BD, Koren S, Phillippy AM. The Harvest suite for rapid core-genome alignment and visualization of thousands of intraspecific microbial genomes. Genome Bio/2014; 15:

17. De Maio N, Wu CH, Wilson DJ. SCOTTI: Efficient Reconstruction of Transmission within Outbreaks with the StructuredCoalescent. PLoS Comput Biol 2016; 12:

18. Chmielarczyk A, Higgins PG, Wojkowska-Mach J, Synowiec E, Zander E, Romaniszyn D, et al. Control of an outbreak of Acinetobacter baumannii infections using vaporized hydrogen peroxide. J Hosp Infect 2012; 81: 239-245.

19. Ou HY, Kuang SN, He X, Molgora BM, Ewing PJ, Deng Z, et al. Complete genome sequence of hypervirulent and outbreak-associated Acinetobacter baumannii LAC-4: epidemiology, resistance genetic determinants and potential virulence factors. Sci Rep 2015; 5:

20. Jones CL, Clancy M, Honnold C, Singh S, Snesrud E, Onmus-Leone F, et al. Fatal outbreak of an emerging clone of extensively drug-resistant Acinetobacter baumannii with enhanced virulence. Clin Infect Dis 2015; 61:145-154.

21. Kaiser T, Finstermeier K, Hantzsch M, Faucheux S, Kaase M, Eckmanns T, et al. Stalking a lethal superbug by whole-genome sequencing and phylogenetics: in- fluence on unraveling a major hospital outbreak of carbapenem-resistant Kleb- siella pneumoniae. Am J Infect Control 2017; 46: 54-59. $\nabla$

22. Kwong JC, Lane CR, Romanes F, Goncalves da Silva A, Easton M, Cronin K, et al. Translating genomics into practice for real-time surveillance and response to carbapenemase-producing Enterobacteriaceae: evidence from a complex multi-institutional KPC outbreak. PeerJ 2018; 6:

23. van Duin D, Perez F, Rudin SD, Cober E, Hanrahan J, Ziegler J, et al. Surveillance of carbapenemresistant Klebsiella pneumoniae: tracking molecular epidemi- ology and outcomes through a regional network. Antimicrob Agent Chemother 2014; 58: 4035-4041.

24. Snitkin ES, Won S, Pirani A, Lapp Z, Weinstein RA, Lolans K, et al. Integrated genomic and interfacility patient-transfer data reveal the transmission pathways of multidrug-resistant Klebsiella pneumoniae in a regional outbreak. Sci Trans Med 2017; 9: $邓$ 
25. Worby CJ, Lipsitch M, Hanage WP. Within-host bacterial diversity hinders accurate reconstruction of transmission networks from genomic distance data. PLoS Comput Biol 2014; 10:

26. Didelot X, Gardy J, Colijn C. Bayesian inference of infectious disease transmission from. wholegenome sequence data. Mol Biol Evol 2014; 31: 1869-1879.

27. Hall M, Woolhouse M, Rambaut A. Epidemic reconstruction in a phylogenetics framework: transmission trees as partitions of the node set. PLoS Comput Biol 2015; 11:

28. Romero-Severson EO, Bulla I, Leitner T. Phylogenetically resolving epidemiologic linkage. Proc Natl Acad Sci U S A 2016; 113: 2690-2695.

29. De Maio N, Wu CH, O'Reilly KM, Wilson D. New Routes to Phylogeography: A Bayesian Structured Coalescent Approximation. PLoS Genet 2015; 11:

30. De Maio N, Wu CH, Wilson DJ. SCOTTI: Efficient Reconstruction of Transmission within Outbreaks with the Structured Coalescent. PLoS Comput Biol2016; 12:

\section{Figures}

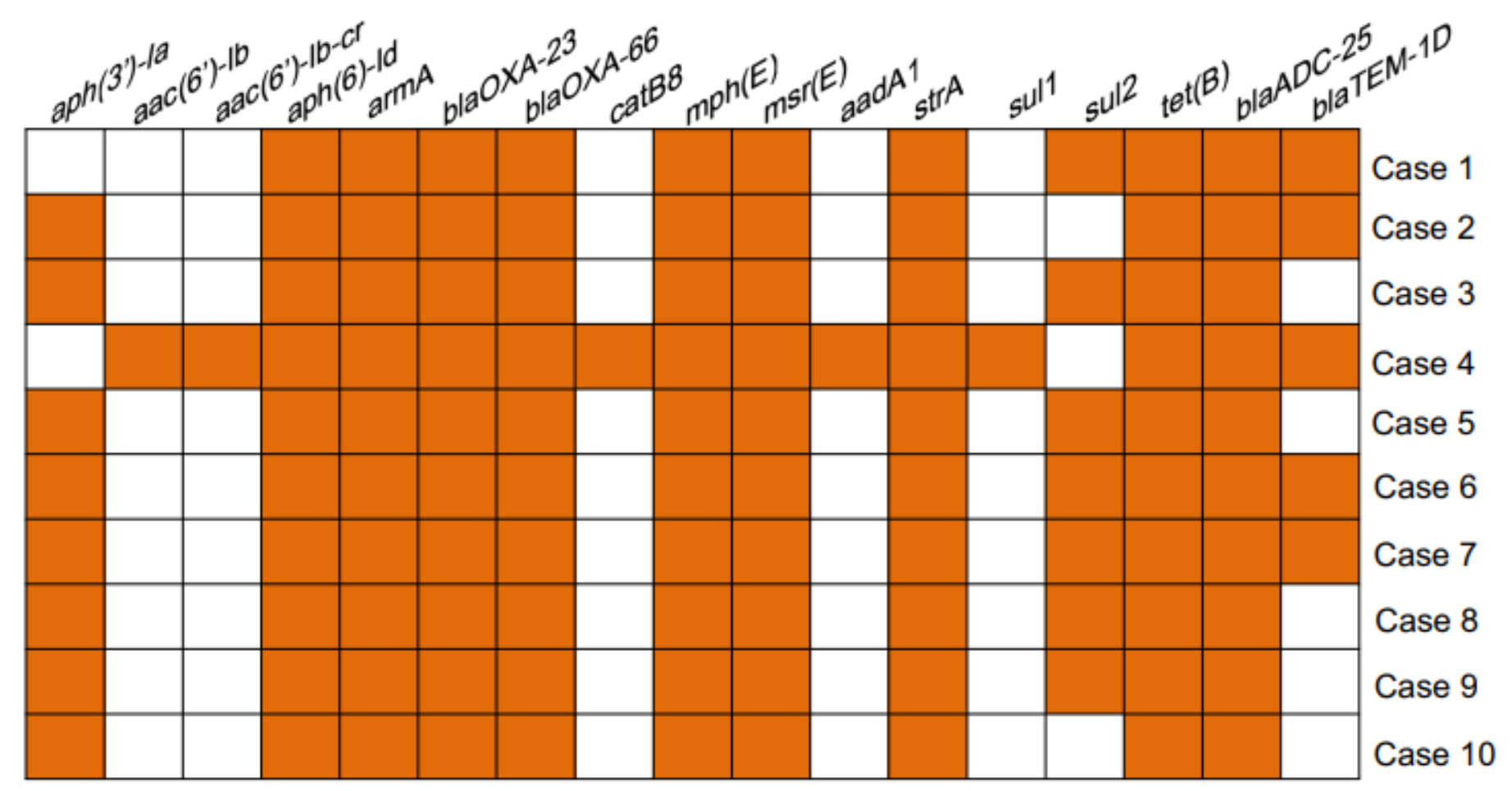

Figure 1

Antimicrobial resistance genes. 

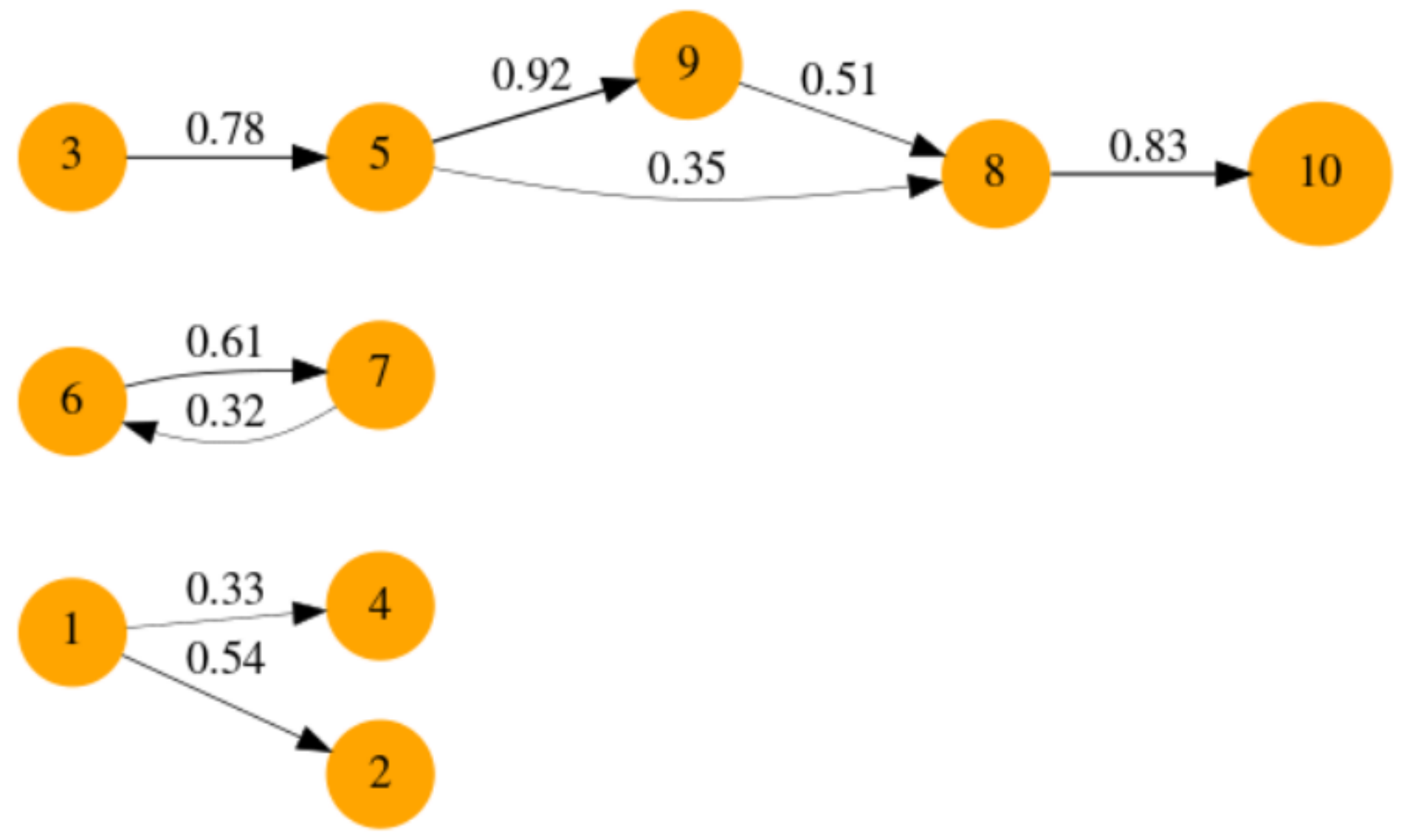

Figure 2

The transmission network of isolates in this study. Numbers on arrows represent the inferred probabilities of the corresponding transmission events. Probabilities bigger than $20 \%$ were shown. 


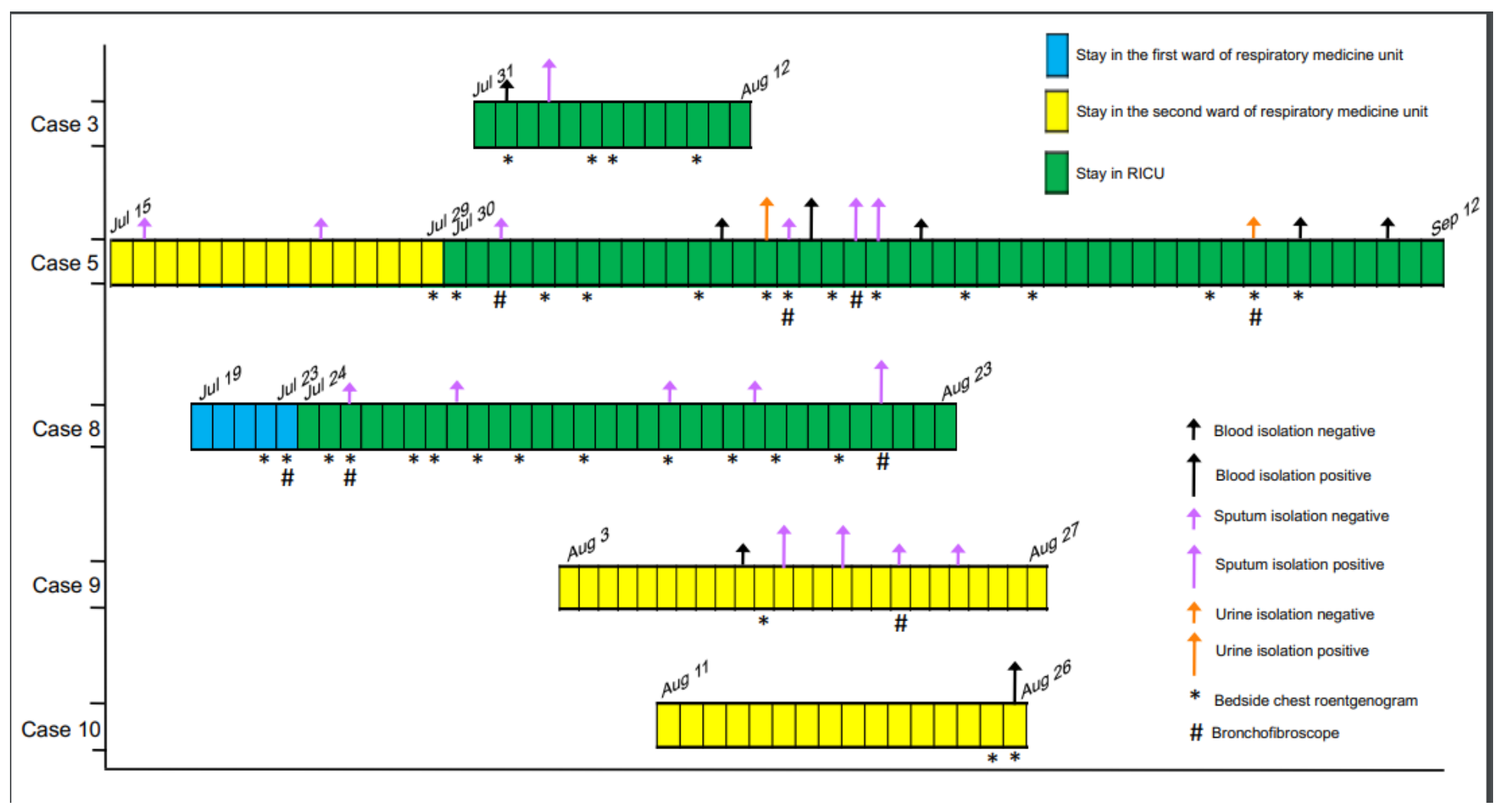

Figure 3

Epidemiology of Acinetobacter baumannii outbreak cases. The dates represent the admission, transference, and discharge of the patient.

\section{$2.5 \times 10^{8} \mathrm{CFU}$}

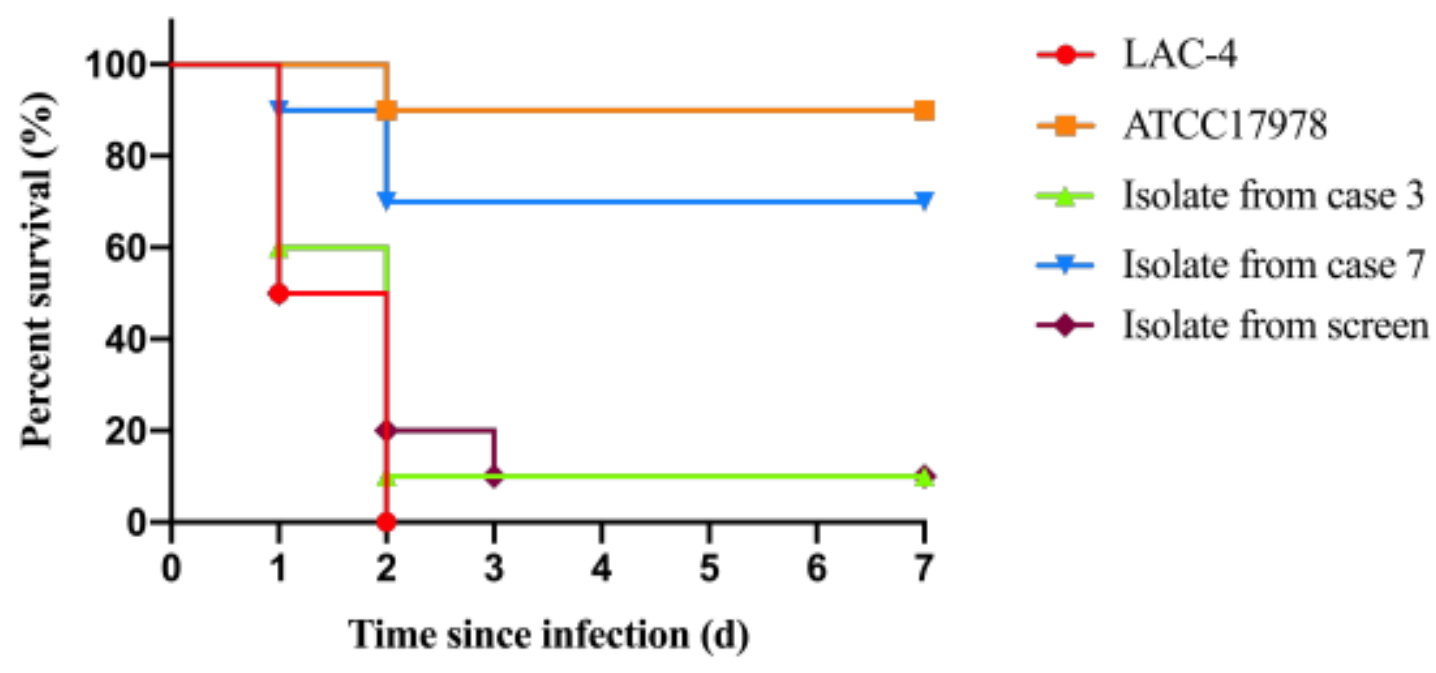

Figure 4

Virulence potential of Acinetobacter baumannii isolates in a mouse infection model. 